\title{
Presence of the Jehol Biota turtle Ordosemys liaoxiensis in the Early Cretaceous Hengtongshan Formation of southern Jilin Province, China
}

\author{
Chang-Fu Zhou ${ }^{1}$, Wen-Hao Wu ${ }^{2,3}$, and Márton Rabi ${ }^{4,5}$ \\ ${ }^{1}$ College of Earth Science and Engineering, Shandong University of Science and Technology, \\ Qingdao 266590, Shandong, China \\ ${ }^{2}$ Key Laboratory for Evolution of Past Life and Environment in Northeast Asia, Ministry of Education, \\ Changchun 130026, Jilin, China \\ ${ }^{3}$ Research Center of Palaeontology and Stratigraphy, Jilin University, Changchun 130056, Jilin, China \\ ${ }^{4}$ Central Natural Science Collections, Martin Luther University Halle-Wittenberg, Halle (Saale), Germany \\ ${ }^{5}$ Department of Geosciences, University of Tübingen, Tübingen, Germany
}

Correspondence: Chang-Fu Zhou (zhoucf528@163.com) and Wen-Hao Wu (wenhao.wu @ foxmail.com)

Received: 25 May 2019 - Revised: 15 August 2019 - Accepted: 19 August 2019 - Published: 12 September 2019

\begin{abstract}
Recently, a vertebrate assemblage of the Jehol Biota has been reported from the Early Cretaceous Hengtongshan Formation of Xingling Town, Meihekou City, Jilin Province, China. It is dominated by the fishes Lycoptera and Sinamia and the sinemydid turtle Ordosemys. Here, we describe the turtle specimens and referral to Ordosemys liaoxiensis, otherwise known from the older Yixian Formation of the Jehol Biota. It is characterized by a subcircular shell, wide vertebral scales, well-developed plastral fenestrae, and a major contribution from the xiphiplastra to enclose the hypo-xiphiplastral fenestra. As the first Mesozoic turtle of Jilin Province, this record represents the first tetrapod to indicate the presence of the Jehol Biota in the region. Given the geographic and temporal distance from the Yixian Formation, future collections from the Hengtongshan Formation have good potential for evaluating spatiotemporal patterns of the Jehol Biota.
\end{abstract}

\section{Introduction}

The Jehol Biota of northeastern China (western Liaoning and conjoint areas) is renowned for providing exceptional insights into an Early Cretaceous ecosystem through its exquisite preservation of plant, invertebrate, and vertebrate fossils (e.g., Zhou et al., 2003; Pan et al., 2013). In the field, the Jehol Biota is typically recognized by the pres- ence of the Eosestheria (crustacean)-Ephemeropsis trisetalis (mayfly insect)-Lycoptera (fish) community (T. Shao et al., 2017). Freshwater sinemydid turtles are among the most common tetrapod fossils of the Jehol Biota, which makes their biostratigraphic potential of interest (e.g., Ji, 1995; Tong et al., 2004; Zhou, 2010a, b; Zhou and Rabi, 2015; S. Shao et al., 2017, 2018; Li et al., 2019). However, these fossil turtles have been less commonly collected outside the typical western Liaoning area of the Jehol Biota (e.g., Ma, 1986; Li et al., 2015; Li and Tong, 2017; Wu et al., 2018). Recently, as the first record of the Mesozoic turtles from Jilin Province, the sinemydid Ordosemys has been reported from the Early Cretaceous Hengtongshan Formation of Xingling Town, Meihekou City (Wu et al., 2018). This material has not been described yet, and its species-level taxonomy awaits clarification. In this study, we describe this material, together with other two specimens, and clarify their taxonomic status. We show that these turtles represent the first tetrapod evidence for the presence of the Jehol Biota in the Hengtongshan Formation. Previous studies correlated the Hengtongshan Formation with the Jiufotang Formation of the Jehol Biota of western Liaoning and conjoint areas based on radiometric dating (119-123 Myr; Y. Chen et al., 2008; Sui and Chen, 2012; J. Chen et al., 2016) and biostratigraphic data (e.g., Lycoptera, Ephemeropsis, Eosestheria, Huashia, Diestheria, and Orthestheria) (e.g., JBGMR, 1982; Chang et al., 2009; Wu et al., 2018). 
The sinemydid turtle Ordosemys has a broad paleogeographic distribution in northern China (e.g., Danilov and Parham, 2007; Li et al., 2019). Three species are widely accepted, including the type species Ordosemys leios from the Ordos Basin of Inner Mongolia and Mengyin Basin of Shandong (Brinkman and Peng, 1993; Brinkman and Wu, 1999; Li et al., 2019), O. liaoxiensis from the Yixian Formation of western Liaoning (e.g., Ji, 1995; Tong et al., 2004), and $O$. brinkmania from Junggar Basin of Xinjiang (Danilov and Parham, 2007). Ordosemys donghai (i.e., Manchurochelys donghai, Ma, 1986) as recombined by Brinkman et al. (2008) from Heilongjiang, is possibly excluded from Ordosemys in lacking the typical generic features ( $\mathrm{Li}$ et al., 2019).

\section{Geographic and geologic settings}

The fossil site is located at a small quarry near Shuanghe Village, Xingling Town, approximately $15 \mathrm{~km}$ southeast of the Meihekou City, southern Jilin Province, China $\left(42^{\circ} 24^{\prime} 54.52^{\prime \prime} \mathrm{N}, 125^{\circ} 51^{\prime} 02.21^{\prime \prime} \mathrm{E}\right.$; Fig. 1). Five fossilbearing layers can be identified in the outcrop of the fossil site (Fig. 1b). The turtle fossils described in this study are from the lower layer, associated with the fishes Lycoptera davidi and Sinamia sp. However, as described by the local farmers, an upper layer is a possible second one bearing fossil turtles. Unfortunately, the fossil turtles were lost by the local farmers.

The fossil-bearing beds are mainly composed of lacustrine deposits of thick layers of greyish-green sandstones and siltstones, which belong to the middle part of the Hengtongshan Formation (JBGMR, 1982; Wu et al., 2018). The Hengtongshan Formation is well exposed in southern Jilin Province and spans 119-123 Myr based on radiometric dating (e.g., Chen et al., 2008, 2016; Sui and Chen, 2012). Based on this, the Hengtongshan Formation is roughly coeval to the Jiufotang Formation as also suggested by biostratigraphic data (e.g., Lycoptera, Ephemeropsis, Eosestheria, Huashia, Diestheria, and Orthestheria) (e.g., JBGMR, 1982; Chang et al., 2009; Wu et al., 2018).

\section{Samples and methods}

Three turtle specimens and associated fishes Lycoptera and Sinamia were collected by the local farmers from a quarry near Shuanghe Village, Xingling Town, Meihekou City, southern Jilin Province, China (Fig. 1). The fossils are deposited in the Paleontological Collection of Xingling Town. Before this study, a skillful preparation of three specimens was performed in the Research Center of Palaeontology and Stratigraphy, Jilin University. The fossils are deposited in greyish-green sandstones and are strongly compressed dorsoventrally. The largest specimen (XL-009; Fig. 2) preserves much of the plastron with several fragments and traces of the carapace; the full ossification of the carapace indicates an adult stage for this specimen. The other two specimens (XL-007 and XL-008; Figs. 3-4) are slightly smaller with XL-008 exhibiting large costo-peripheral fenestrae, whereas XL-007 reduced to closed costo-peripheral fenestrae. The three specimens are identified as the same taxon representing different ontogenetic stages.

\section{Institution abbreviations}

IVPG, Institute of Vertebrate Paleontology, Gansu Agricultural University, Lanzhou, China; GSGM, Gansu Geological Museum, Lanzhou, China; PMOL, Paleontological Museum of Liaoning, Shenyang Normal University, Shenyang, China; XL, Paleontological Collection of Xingling Town, Meihekou, China.

\section{Systematic paleontology}

Testudinata Klein, 1760

Testudines Batsch, 1788

Pan-Cryptodira Joyce, Parham \& Gauthier, 2004

Sinemydidae sensu Rabi, Sukhanov, Egorova, Danilov \& Joyce, 2014

Ordosemys liaoxiensis (Ji 1995)

(Figs. 2-4)

\section{Specimens}

XL-007 (Fig. 3), a subadult specimen with most of the shell; XL-008 (Fig. 4), a juvenile skeleton including a partial skull, cervicals, shell and forelimbs; XL-009 (Fig. 2), an adult skeleton including a partial carapace, much of the plastron, and some appendicular bones. These fossils are deposited in the Paleontological Collection of Xingling Town (XL).

\section{Locality and horizon}

A quarry (GPS: $42^{\circ} 24^{\prime} 54.52^{\prime \prime} \mathrm{N}, 125^{\circ} 51^{\prime} 02.21^{\prime \prime} \mathrm{E}$ ) near Shuanghe Village, Xingling Town, Meihekou City, southern Jilin Province, China (Fig. 1); the Early Cretaceous Hengtongshan Formation (119-123 Myr).

\section{Description}

\subsection{Osteology of XL-009}

XL-009 is the largest specimen with an estimated plastral width of $144 \mathrm{~cm}$. The carapace is mostly missing, but the imprints reveal that the costo-peripheral fenestrae were fully ossified (Fig. 2a, b). Neurals 1 and 2 are partially preserved together with the sulcus of vertebrals 1-2 across neural 1 and costal 1 . The sulcus is slightly protruding anteriorly, which is 


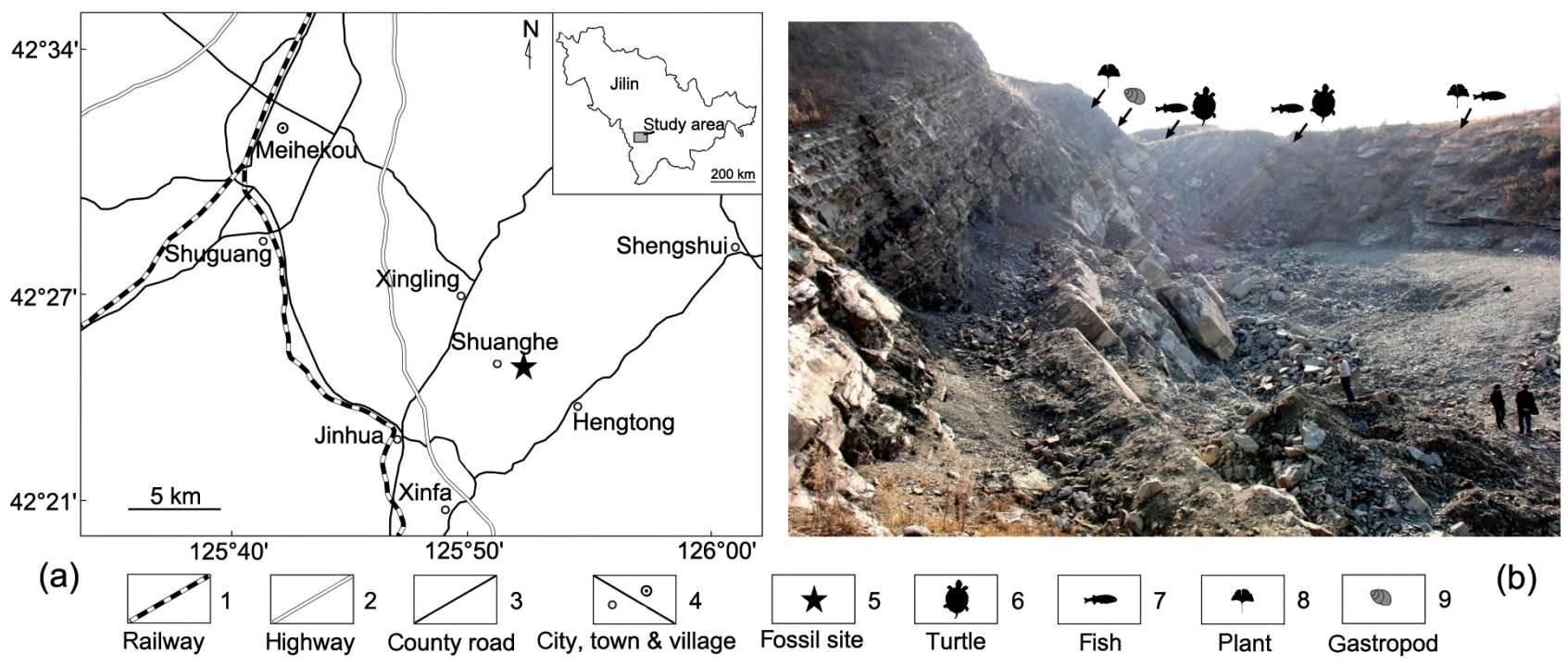

Figure 1. (a) Fossil locality (asterisk; $42^{\circ} 24^{\prime} 54.52^{\prime \prime} \mathrm{N}, 125^{\circ} 51^{\prime} 02.21^{\prime \prime} \mathrm{E}$ ) of Ordosemys liaoxiensis from the Early Cretaceous Hengtongshan Formation of Shuanghe Village, Xingling Town, Meihekou City, Jilin Province, northeastern China. (b) Outcrop of the fossil site showing five fossil-bearing layers; the turtle specimens described in this study are from the lower layer.

also known for Ordosemys leios and O. brinkmania (Danilov and Parham, 2007; Li et al., 2019). The preneural plate is typical for Ordosemys spp., and its suture with neural 1 is generally positioned behind the sulcus of vertebrals $1-2$ in Ordosemys spp. (e.g., Brinkman and Peng, 1993; Tong et al., 2004; Danilov and Parham, 2007; Li et al., 2019), or confluent with the sulcus of the vertebrals 1-2 (a juvenile of $O$. leios; IVPG-T001-2; Li et al., 2019). However, the suture is uncertain in the corresponding region of XL-009.

The plastron is well exposed with the hyoplastron, hypoplastron, and xiphiplastron in visceral view (Fig. 2c, d). The plastral fenestrae are open, including the two medial and two lateral fenestrae, as in Ordosemys leios, O. liaoxiensis, and Liaochelys jianchangensis (Brinkman and Peng, 1993; Tong et al., 2004; Zhou, 2010a). The central and lateral plastral fenestrae are enlarged in size, whereas the hypoxiphiplastral fenestra is much smaller. The xiphiplastra contributes to the hypo-xiphiplastral fenestra to a greater extent than the hypoplastra. In contrast, the fenestra is enclosed by the xiphiplastra and hypoplastra subequally in $O$. leios, O. liaoxiensis, and L. jianchangensis (Brinkman and Peng, 1993; Tong et al., 2004; Zhou, 2010a).

The right hyoplastron is $72 \mathrm{~mm}$ in width. The hyoplastron and hypoplastron are firmly articulated and form the plastral bridge. The bridge is $45 \mathrm{~mm}$ in length. Laterally, the hyoplastron and hypoplastron are characteristic of the peg-like processes which articulated with the carapace through ligaments. The hypoplastra enclosed the posterior medial fenestra with the xiphiplastra. The xiphiplastra is largely covered by the pelvic girdle. The xiphiplastron seems to be elongated as in Ordosemys spp. but different from the condition seen in Liaochelys jianchangensis.
The pectoral girdle is only preserved with the two straplike coracoids. The right pelvic girdle is exposed with the pubis and ischium. The pubis is triradiate with the lateral pubic process, the medial pectineal process, and the dorsal acetabular process. The lateral pubic process is comparable with the pectineal process in size. The acetabular process is stronger and firmly articulates with ischium. The right femur is preserved in articulation with the acetabulum and is about $40 \mathrm{~mm}$ long. The tibia and fibula are only preserved with their proximal ends.

\subsection{Osteology of XL-007}

The carapace appears to be subcircular with an estimated width of $130 \mathrm{~mm}$ across costal 4 (Fig. 3). The carapace is smooth and sculptured by the scale sulci. Costals $2-8$ are preserved and the costo-peripheral fenestrae seem to be open between costals 2-4 and peripherals $4-7$, but the posterior ones are fully closed. In contrast, the similar region is completely ossified in the larger specimen XL-009, based on the imprints of the carapace (Fig. 2a, b). Therefore, the open condition implies that XL-007 represents a subadult individual.

Distally, the rib ends are exposed similarly to the condition in other sinemydids. Costal 3 is not expanded distally, different from the condition of Liaochelys jianchangensis (Zhou, 2010a).

The sulci of vertebral and pleural scales are deep and identifiable. The lateral termination of vertebral 3 can be traced. Its width is about $70 \mathrm{~mm}$, more than half the width of the carapace indicating that the pleural is narrower than the vertebral as also seen in Ordosemys spp., Liaochelys jianchangensis, and Xiaochelys ningchengensis (e.g., Brinkman 


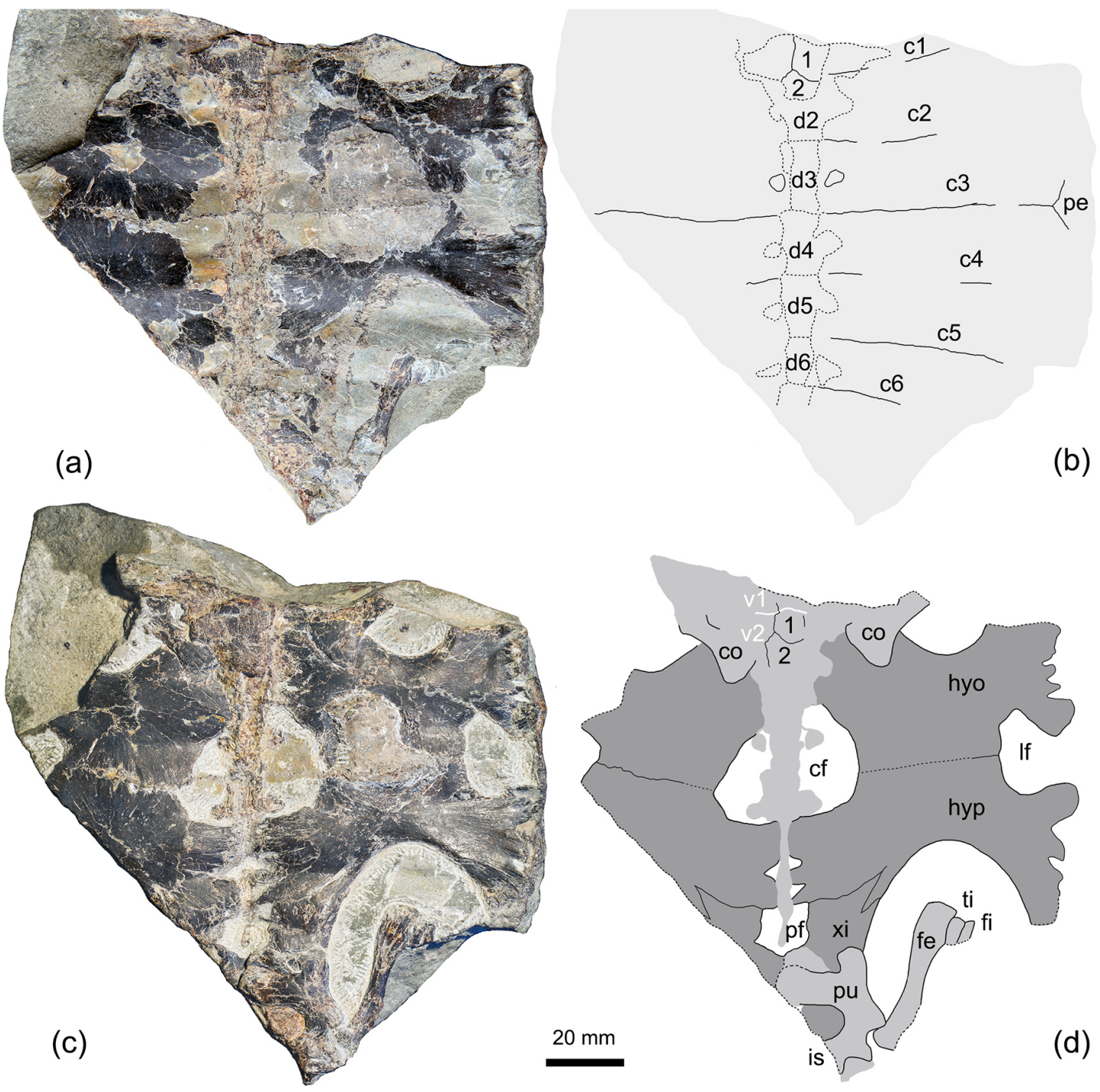

Figure 2. Ordosemys liaoxiensis (XL-009; most in visceral view) from the Early Cretaceous Hengtongshan Formation of Shuanghe Village, Xingling Town, Meihekou City, Jilin Province, northeastern China. (a, b) Carapace before preparation; (c, d) plastron and appendicular elements after preparation. Abbreviations: 1-2, neural plates 1-2; c1-c6, costal plates 1-6; cf, central plastral fenestra; co, coracoid; d2-d6, dorsal vertebrae 2-6; fe, femur; fi, fibula; hyo, hyoplastron; hyp, hypoplastron; is, ischium; lf, lateral plastral fenestra; pe, peripheral plate; pf, posterior medial plastral fenestra; pu, pubis; ti, tibia; v1-v2, vertebral scales 1-2; xi, xiphiplastron.

and Peng, 1993; Tong et al., 2004; Danilov and Parham, 2007; Zhou, 2010a; Zhou and Rabi, 2015).

The plastron has a limited exposure with the inguinal buttress of the hypoplastron. The inguinal buttress terminates at peripheral 8 .

\subsection{Osteology of XL-008}

$\mathrm{XL}-008$ is a juvenile skeleton and is exposed dorsally (Fig. 4). The partial skull is poorly preserved in articulation with the damaged cervical series. The forelimbs are partially preserved with the distal end of the humeri, the right ulna, and radius. The ulna is about $20 \mathrm{~mm}$ long.
The carapace is also strongly damaged, resulting in the extensive exposure of the plastron in dorsal view. Large costoperipheral fenestrae are present, implying a juvenile stage for this specimen. The carapace shows a subcircular profile. The preserved length is about $120 \mathrm{~mm}$, but the estimated length is about $130 \mathrm{~mm}$. The maximum width is about $130 \mathrm{~mm}$ and comparable with that of XL-007. The cervical scale is wide. Other sulci are obscured due to the broken surface of the carapace. The nuchal emargination is moderately developed.

The hyoplastron and hyoplastron are enclosing the large central fenestra and lateral fenestrae. The bridge is about $34 \mathrm{~mm}$ long. 


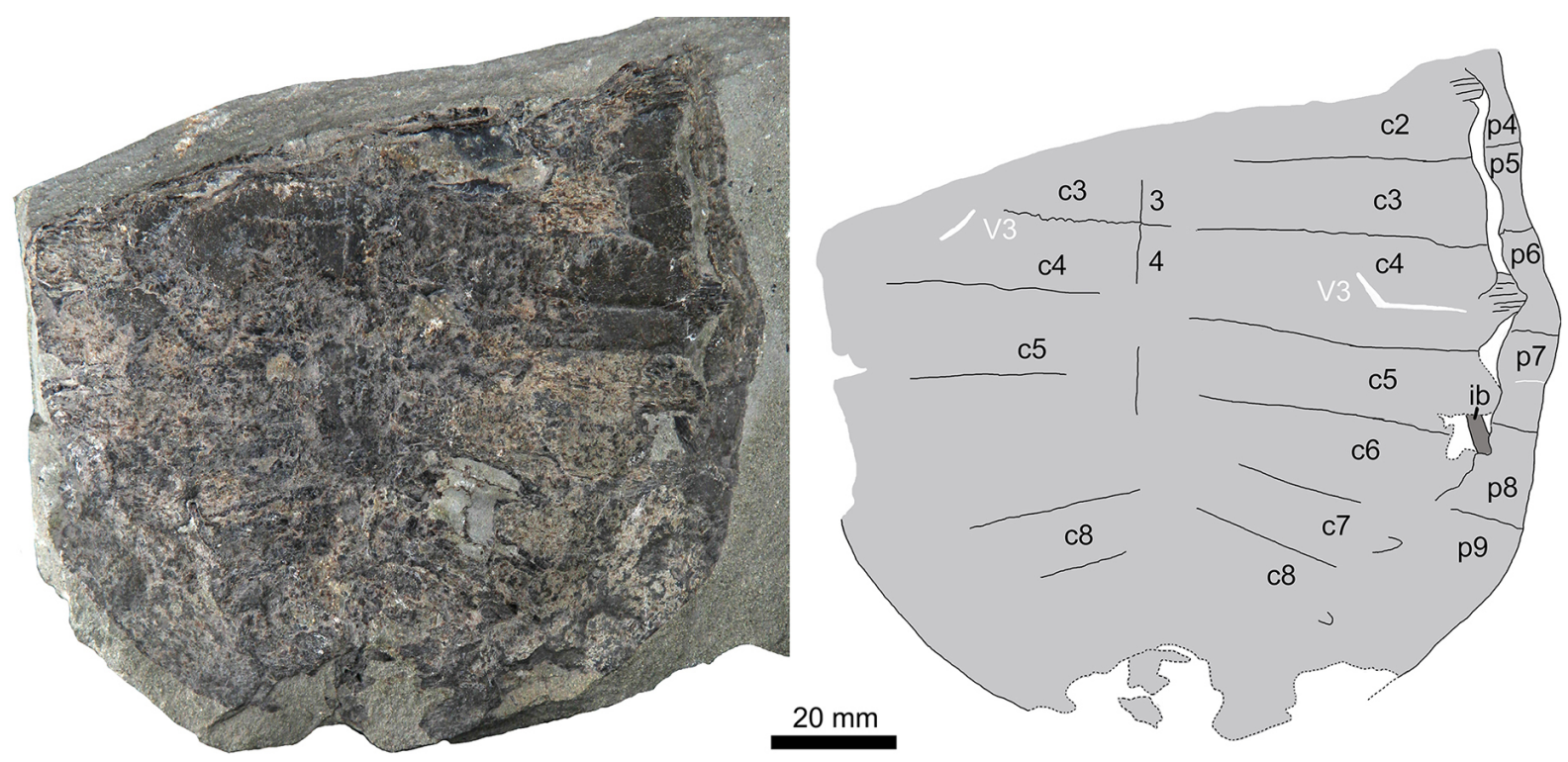

Figure 3. Ordosemys liaoxiensis (XL-007; in dorsal view) from the Early Cretaceous Hengtongshan Formation of Shuanghe Village, Xingling Town, Meihekou City, Jilin Province, northeastern China. Abbreviations: 3-4, neural plates 3-4; c2-c8, costal plates 2-8; ib, inguinal buttress of the hypoplastron; p4-p9, peripheral plates 4-9; v3, vertebral scale 3.

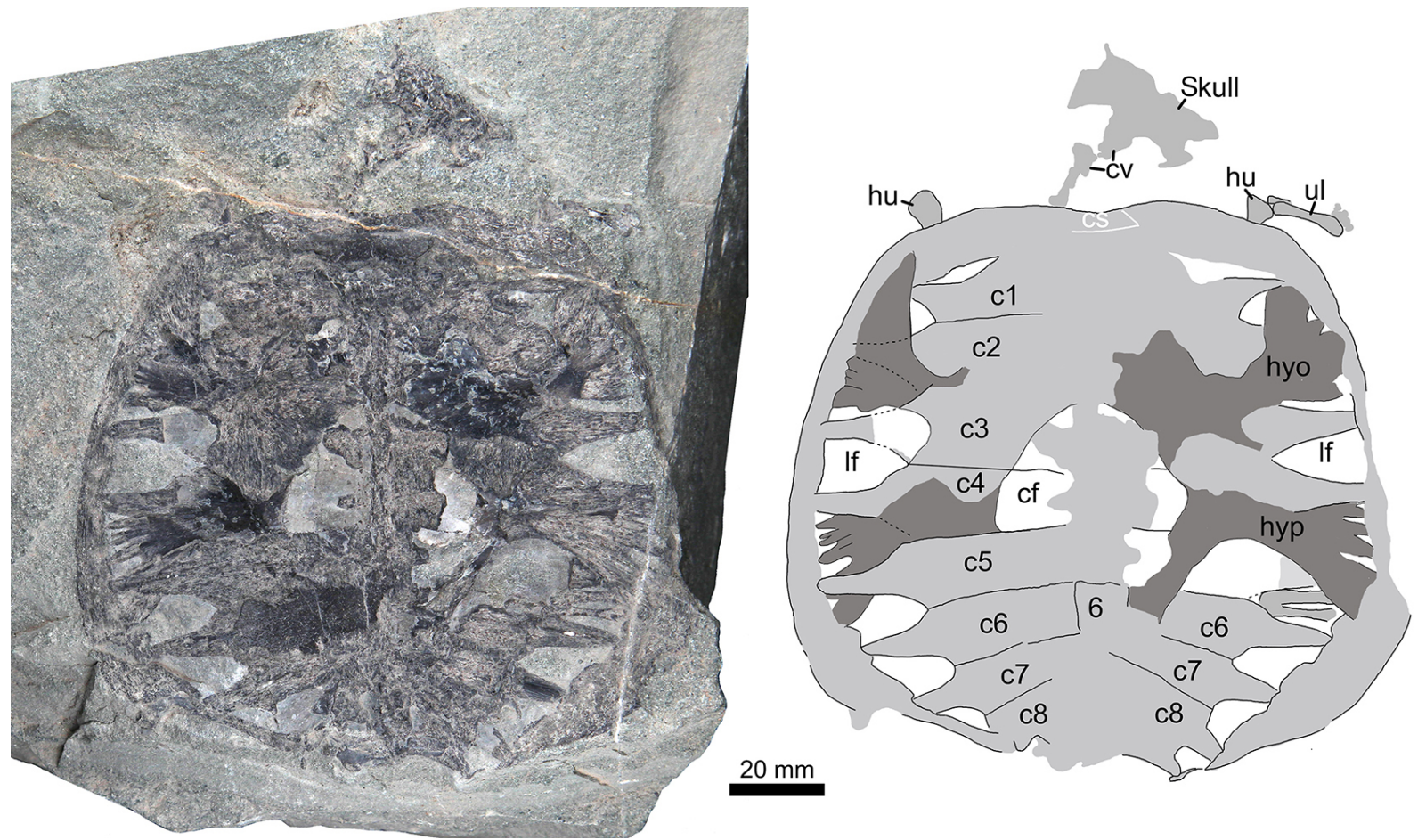

Figure 4. Ordosemys liaoxiensis (XL-008; in dorsal view) from the Early Cretaceous Hengtongshan Formation of Shuanghe Village, Xingling Town, Meihekou City, Jilin Province, northeastern China. Abbreviations: 6, neural plate 6; c1-c8, costal plates 1-8; cf, central plastral fenestra; cs, cervical scale; cv, cervical vertebrae; hu, humerus; hyo, hyoplastron; hyp, hypoplastron; lf, lateral plastral fenestra; pe, peripheral plates; ul, ulna. 


\section{Discussion}

\subsection{Taxonomy}

The three specimens represent an ontogenetic series from juvenile (XL-008), to subadult (XL-007), and to adult (XL009) stages. As they show no distinct morphological differences besides the ontogenetic variance and were collected from the same locality and horizon, we refer them to the same taxon. The specimens are similar to Ordosemys spp. and differ from other sinemydids in having a subcircular shell, wide vertebral scales, and large central and lateral fenestrae of plastron (e.g., Brinkman et al., 2008; Li and Tong, 2017; Li et al., 2019). The presence of a preneural plate is also diagnostic for Ordosemys spp. (e.g., Brinkman et al., 2008; Li et al., 2019), but it is uncertain in all three specimens due to the poor preservation.

Three species are widely accepted in the genus Ordosemys: O. leios, O. liaoxiensis, and O. brinkmania (Danilov and Parham, 2007; Brinkman et al., 2008; Li and Tong, 2017; Li et al., 2019). Two further species, O. perforata (i.e., Asiachelys perforata sensu Sukhanov and Narmandakh, 2006; Danilov and Parham, 2007) and O. donghai (i.e., Machurochelys donghai sensu Ma, 1986; Brinkman et al., 2008; Li and Tong, 2017), have been questioned in their affinities to Ordosemys in lacking the preneural plate and wide vertebral scales (e.g., Li et al., 2019).

The Jilin specimens differ from $O$. brinkmania in the presence of a hypo-xiphiplastral fenestra. The fenestra is also present in $O$. leios and $O$. liaoxiensis, but $O$. leios lacks lateral plastral fenestrae. In the former two species, the hypo-xiphiplastral fenestra is enclosed by the hypoplastra and xiphiplastra subequally. In the adult XL-009, however, the xiphiplastra have a major contribution relative to the hypoplastra in enclosing the fenestra (Fig. 2). The hypoxiphiplastral fenestra is also known in other sinemydids including Liaochelys jianchangensis (the adult specimen PMOL-AR00140; Zhou, 2010a), Changmachelys bohlini (the subadult specimen GSGM 05-CM-016, but uncertain in adult specimen GSGM 05 IG-02-0902; Brinkman et al., 2013), and Jeholochelys lingyuanensis (the juvenile specimen PMOL-AR00213, but absent in adult specimens; Shao et al., 2018). The fenestra is therefore subject to ontogenetic variation although the hypo-xiphiplastral fenestra is always enclosed subequally by the hypoplastra and xiphiplastra in these sinemydids. The condition in the adult XL-009 is therefore unusual, but there is still much left to be learned about the ontogeny and individual morphological variation of sinemydids (Shao et al., 2018). Given that the Jilin material only differs from $O$. liaoxiensis in this single character, we refer all specimens to this species.

\subsection{Paleobiogeography of Ordosemys}

Among freshwater sinemydid turtles, Ordosemys spp. is considered to have the broadest paleobiogeographic distribution in northern China (e.g., Danilov and Parham, 2007; Li et al., 2019). The type species Ordosemys leios is from the Ordos Basin of Inner Mongolia (Brinkman and Peng, 1993; see also Brinkman and $\mathrm{Wu}, 1999$ ) and has been recently reported from the Mengyin Basin of Shandong (Li et al., 2019). The second species, $O$. liaoxiensis, is known from the lacustrine deposits of Yixian Formation of Beipiao, western Liaoning (e.g., Ji, 1995; Tong et al., 2004). Ordosemys brinkmania comes from the fluvial deposits of the Tugulu Formation of Wuerho, eastern Xinjiang (Danilov and Parham, 2007). Ordosemys donghai (i.e., Manchurochelys donghai sensu Ma, 1986; Brinkman et al., 2008; Li and Tong, 2017) from Helongjiang was previously considered the easternmost record of Ordosemys in northeastern China (Danilov and Parham, 2007), but this taxon may not belong to Ordosemys because of the lack of a preneural and wide vertebral scales (e.g., $\mathrm{Li}$ et al., 2019). Ordosemys liaoxiensis is so far restricted to northeastern China and the Yixian Formation of the Jehol Biota. The new record expands the distribution of this species from western Liaoning to southern Jilin. This occurrence thus furthermore supports that Ordosemys spp. may have had a broader paleogeographic distribution compared to other sinemydid turtles. As the first Mesozoic turtle of Jilin Province, Ordosemys liaoxiensis from the Early Cretaceous Hengtongshan Formation represents the first tetrapod to indicate the presence of the Jehol Biota in the region. It also extends the stratigraphic range of $O$. liaoxiensis, which was previously only known from the lower Yixian Formation and has not been reported from the overlying Jiufotang Formation of Liaoning, the equivalent of the Hengtongshan Formation of Jilin Province (e.g., Wu et al., 2018). This is likely due to a sampling bias because we are aware of unpublished specimens from the Jiufotang Formation that resemble $\mathrm{Or}$ dosemys spp. Given the morphological difference from the typical $O$. liaoxiensis from the Yixian Formation and given the geographic and age difference, future collections in the Hengtongshan Formation have good potential for evaluating spatial patterns of the Jehol Biota.

Data availability. Three specimens of Ordosemys described in this study are stored in Paleontological Collection of Xingling Town, Meihekou City, Jilin Province, China (accession numbers: XL-007, XL-008, and XL-009). 
Author contributions. CFZ and WHW conceived the project and created figures. CFZ, MR and WHW wrote and approved the paper.

Competing interests. The authors declare that they have no conflict of interest.

Acknowledgements. We would like to thank the director of the Research Center of Palaeontology and Stratigraphy, Jilin University, Chun-Li Sun, for his encouragement. Juliana Sterli and an anonymous reviewer are thanked for their helpful comments that improved the quality of this paper.

Financial support. This research has been supported by the Taishan Scholar Program of Shandong Province (grant no. tsqn201812070), the Shandong Provincial Natural Science Foundation (grant no. ZR2017MD031), and the Research Fund of Shandong University of Science and Technology (grant no. 2015TDJH101) to Chang-Fu Zhou. Maton Rabi received funding from the Volkswagen Foundation (grant no. 90 978).

Review statement. This paper was edited by Florian Witzmann and reviewed by Juliana Sterli and one anonymous referee.

\section{References}

Batsch, A. J. G. C.: Versuch einer Anleitung, zur Kenntniÿund Geschichte der Thiere und Mineralien, Akademische Buchhandlung, Jena, 53-59, 1788.

Brinkman, D. B. and Peng, J.-H.: Ordosemys leios, n. gen., n. sp., a new turtle from the Early Cretaceous of the Ordos Basin, Inner Mongolia, Can. J. Earth Sci., 30, 2128-2138, 1993.

Brinkman, D. B. and Wu, X.-C.: The skull of Ordosemys, an Early Cretaceous turtle from Inner Mongolia, People's Republic of China, and the interrelationships of Eucryptodira (Chelonia, Cryptodira), Paludicola, 2, 134-147, 1999.

Brinkman, D. B., Li, J.-L., and Ye, X.-K.: Order Testudines, in: The Chinese fossil reptiles and their kin, edited by: Li, J. L., Wu, X. C., and Zhang, F. C., Science Press, Beijing, 35-102, 2008.

Brinkman, D. B., Yuan, C.-X., Ji, Q., Li, D.-Q., and You, H.-L.: A new turtle from the Xiagou Formation (Early Cretaceous) of Changma Basin, Gansu Province, P. R. China. Palaeobiol. Palaeoenv., 93, 367-382, 2013.

Chang, S.-C., Zhang, H., Renne, P. R., and Fang, Y.: High-precision ${ }^{40} \mathrm{Ar} /{ }^{39} \mathrm{Ar}$ age for the Jehol Biota, Palaeogeogr. Palaeocl., 280, 94-104, 2009.

Chen, J., Li, B., Yao, Y., Liu, M., Yang, F., Xing D., Li, W., and Wang, Y.: Comparison between Mesozoic volcanic rock strata in northeast of Liaoning-south of Jilin and Yixian Formation in west of Liaoning, Acta Geol. Sin.-Engl., 90, 2733-2746, 2016 (in Chinese with English abstract).

Chen, Y., Sun, C., Sun, Y., and Sun, W.: Cretaceous volcanic events in southeastern Jilin Province, China: evidence from single zircon U-Pb ages, Acta Geol. Sin.-Engl., 82, 1194-1200, 2008.
Danilov, I. G. and Parham, J. F.: The type series of "Sinemys" wuerhoensis, a problematic turtle from the Lower Cretaceous of China, includes at least three taxa, Palaeontology, 50, 431-444, 2007.

Ji, S.-A.: Reptiles, in: Fauna and Stratigraphy of JurassicCretaceous in Beijing and the Adjacent Areas, edited by: Ren, D., Lu, L.-W., Guo, Z.-G., and Ji, S.-A., Seismic Press, Beijing, 140-146, 1995.

Jilin Bureau of Geology and Mineral Resources (JBGMR): Regional geology of Jilin Province, Geological Publishing House, Beijing, 1, 1-698, 1982 (in Chinese with English abstract).

Joyce, W. G., Parham, J. F., and Gauthier, J. A.: Developing a protocol for the conversion of rank-based taxon names to phylogenetically defined clade names, as exemplified by turtles, J. Paleontol., 78, 989-1013, 2004.

Klein, I. T.: Klassification und kurze Geschichte der Vierf üÿigen Thiere, edited by: Behn, F. D., trans. Jonas Schmidt, Lübeck, 1381, 1760.

Li, D.-Q., Zhou, C.-F., Li, L., Yang, J., Li, L., and Rabi, M.: The sinemydid turtle Ordosemys from the Lower Cretaceous Mengyin Formation of Shandong, China and its implication for the age of the Luohandong Formation of the Ordos Basin, Peer J., 7, e6229, https://doi.org/10.7717/peerj.6229, 2019.

Li, J. and Tong, H.: Parareptilians, Captorhines, and Testudines, in: Amphibians, Reptilians, and Avians: Palaeovertebrata Sinica Volume II, Serial 6, edited by: Li, J. and Zhou, Z., Science Press, Beijing, 92-369, 2017.

Li, L., Tong, H., Gu, W., and Liu, J.: A new trionychid turtle from the Early Cretaceous of Heilongjiang Province, Northeastern China, Cretac. Res., 56, 155-160, 2015.

Ma, Z.-L.: Mesozoic turtle fossil from Donghai Coal Mine of Jixi, Heilongjiang Province, Mus. Res., 14, 109-112, 1986 (in Chinese).

Pan, Y., Sha, J, Zhou, Z., and Fursich, F. T.: The Jehol Biota: Definition and distribution of exceptionally preserved relicts of a continental Early Cretaceous ecosystem, Cretaceous Res., 44, 30-38, 2013.

Rabi, M., Sukhanov, V. B., Egorova, V. N., Danilov, I., and Joyce, W.: Osteology, relationships, and ecology of Annemys (Testudines, Eucryptodira) from the Late Jurassic of Shar Teg, Mongolia, and phylogenetic definitions for Xinjiangchelyidae, Sinemydidae, and Macrobaenidae, J. Vertebr. Paleontol., 34, 327352, 2014.

Shao, S., Yang, Y., Li, L., Sun, D.-Y., and Zhou, C.-F.: The first juvenile specimen of Manchurochelys manchoukuoensis from the Early Cretaceous Jehol Biota, Peer J., 5, e3274, doi:10.7717/peerj.3274, 2017.

Shao, S., Li, L., Yang, Y., and Zhou, C.-F.: Hyperphalangy in a new turtle of the Early Cretaceous Jehol Biota, Peer J., 6, e5371, doi:10.7717/peerj.5371, 2018.

Shao, T., Zhang, H., Wang, Q., Liu, Y., and Zhang, Y.: A Mesozoic Pompeii: History of the Jehol Biota's rise and fall, Acta Geol. Sin.-Engl., 91, 1893-1903, 2017.

Sui, Z.-M. and Chen, Y.-J.: Petrogenesis of volcanic rocks from Sankeyushu Formation in southern Jilin: evidence from Zircon $\mathrm{U}-\mathrm{Pb}$ ages and $\mathrm{Hf}$ isotopes, Geoscience, 26, 627-634, 2012 (in Chinese with English abstract).

Sukhanov, V. B. and Narmandakh, P.: New taxa of Mesozoic turtles from Mongolia, Fossil Turtle Research, 1, 119-127, 2006. 
Tong, H., Ji, S.-A., and Ji, Q.: Ordosemys (Testudines: Cryptodira) from the Yixian Formation of Liaoning Province, northeastern China: new specimens and systematic revision, Am. Mus. Novit., 3438, 1-20, 2004.

Wu, W., Zhou, C., Sun, W., and Sun, C.: The first vertebrate assemblage dominated by fishes and turtles of the Jehol Biota in Jilin Province, NE China, Acta Geol. Sin.-Engl., 92, 2028-2029, 2018.

Zhou, C.-F.: A new eucryptodiran turtle from the Early Cretaceous Jiufotang Formation of western Liaoning, China, Zootaxa, 2676, 45-56, 2010a.
Zhou, C.-F.: A second specimen of Manchurochelys manchoukuoensis Endo \& Shikama, 1942 (Testudines: Eucryptodira) from the Early Cretaceous Yixian Formation of western Liaoning, China, Zootaxa, 2534, 57-66, doi:10.11646/zootaxa.2534.1.4, 2010b.

Zhou, C.-F. and Rabi, M.: A sinemydid turtle from the Jehol Biota provides insights into the basal divergence of crown turtles, Sci. Rep., 5, 16299, doi:10.1038/srep16299, 2015.

Zhou, Z.-H., Barrett, P. M., and Hilton, J.: An exceptionally preserved Lower Cretaceous ecosystem, Nature, 421, 807-814, 2003. 